\title{
Commentary on population screening for fragile $X$ syndrome
}

\author{
Bradford Coffee, PhD
}

1

$\mathrm{n}$ this issue of Genetics in Medicine, Hill et al. ${ }^{1}$ conduct a systematic review of studies investigating population screening for fragile X syndrome (FXS) among two groups: women of reproductive age and newborns. Most of the studies obtained through their review focus on the psychosocial and counseling issues of screening adult women of reproductive age for the FMR1 mutation alleles, with one small pilot study examining newborn screening for FXS. Because of the unique biology of the FMR1 mutation, population screening for FXS raises concerns that are not found in population screening for disorders such as phenylketonuria and cystic fibrosis. For FMR1, two mutation classes are of medical concern: the premutation and the full mutation. Male premutation carriers pass on the premutation to all their daughters and are themselves at risk of developing fragile X-associated tremor/ataxia syndrome (FXTAS) later in life. Female premutation carriers are at risk of having a child with FXS, of having fragile X-associated primary ovarian insufficiency (FXPOI) and of developing FXTAS; although the latter occurs at a much lower rate than in male carriers. Full mutation carrier males almost always have frank intellectual disabilities. The full mutation carried by women, however, is incompletely penetrant and variably expressed. This has led to a concern that identifying full mutation carrier females via population screening programs could lead to stigmatization of clinically unaffected females. For all these reasons, current guidelines recommend that population screening for FXS be limited to well-defined clinical research protocols.

In their review, Hill et al. ${ }^{1}$ find that screening adult women for FMR1 mutations is less controversial than newborn screening for FXS. Among adult women, preconception screening is preferred to screening during pregnancy. As Hill et al. ${ }^{1}$ note, preconception screening would actually serve two purposes: assessment of a woman's risk for having a child with FXS and assessment of her risk for FXPOI. Voluntary screening of adult women for FMRI mutations to assess reproductive risk is generally viewed favorably by women, even among those who chose not to be screened. To date, there has been no evidence that offering voluntary screening to this population has any negative impact. As with any population screening for a genetic condition, family members would be identified as mutation carriers through cascade testing. Unique to FMRI mutations, this also serves as predictive testing for the premutation lateonset disorders of FXPOI and FXTAS, in addition to the risk for offspring with FXS. This raises concerns of the impact on family members who did not consent to being screened. These concerns, however, are similar to those raised in testing for familial cancer predisposition syndromes, such as $B R C A 1$ and $B R C A 2$ testing. Unlike newborn screening, population screen-

From the Department of Human Genetics, Emory University School of Medicine, Atlanta, GA.

Bradford Coffee, PhD, Department of Human Genetics, Emory University School of Medicine, Atlanta, GA 30322. E-mail: bcoffee@emory.edu.

Disclosure: The author declares no conflict of interest

DOI: 10.1097/GIM.0b013e3181e5c804 ing of adult women of reproductive age would be done with their fully informed consent, thereby reducing the negative impact of screening. Access to education and expert genetic counseling services would be needed to help balance the risk to benefit ratio for each unique family.

Turning to population screening for newborns brings up different concerns. The identification of FXS males in the newborn period would offer the opportunity for early educational intervention, prevent the "diagnostic odyssey" of trying to establish a diagnosis in an affected child, and inform couples about their risk of having another child with FXS. However, there are three major concerns regarding screening for FMRI mutations during the newborn period. First, there is no diagnostic test to determine which full mutation carrier females are destined to be affected with FXS, and identifying all full mutation carrier females raises the concern that these girls will be at risk for the "vulnerable child syndrome," where the expectation of disease itself causes disease or worsens subtle manifestations of disease. One way to avoid the vulnerable child syndrome is of course to simply not screen infant girls for FXS; however, excluding girls from screening leads to questions of equity in screening, as full mutation carrier females who are affected with FXS could clearly benefit from early detection, as would males. A second concern is that population screening for FMR1 mutations may result in the incidental identification of infants with sex chromosome aneuploidies. For example, Klinefelter syndrome is approximately eight times more prevalent than FXS in males, whereas Turner syndrome, with a prevalence of about one in 4,000 , has a slightly higher prevalence than full mutation carrier females. Therefore, for every infant identified with the FMRl full mutation, approximately five infants will be identified with a sex chromosome aneuploidy. As with the identification of full mutation carrier females, early identification recognition of sex chromosome aneuploidies can be beneficial. However, stigmatization and the vulnerable child syndrome are also concerns for individuals identified with sex chromosome aneuploidies, because they may show no signs or only mild clinical manifestations of the disease in childhood. Finally, the third concern is the identification of premutation carriers, a significant fraction of whom will be destined to develop adult-onset conditions. Predictive screening of infants for adult-onset conditions is clearly not a goal of newborn screening. Sizing CGG repeats would lead to the detection of premutations, although programmatic decisions could be made not to report premutations. Using aberrant FMRI methylation as a screening tool for FXS circumvents the problem of detecting premutation carriers, as opposed to detection of expanded CGG repeats. Unfortunately, methylation analysis in females cannot accurately predict whether a girl will be affected with FXS. Development of other high-throughput technologies, perhaps, such as quantification of the FMRI protein, might provide a solution for these issues. In addition, as Hill et al. ${ }^{1}$ rightly point out, more studies examining the psychosocial impact of newborn screening are needed in general to better understand the risks and benefits of FMR1 screening. 
One advantage of adding FXS to existing newborn screening programs is this would ensure that virtually every infant in the United States would be screened for FXS. However, there have been recent controversies about newborn screening with some people in the general public distrusting how that information is being used in their child's healthcare. Clearly, better and earlier education needs to be provided to the general public, preferably before birth, to make them aware of the benefits and huge successes of newborn screening. Nevertheless, it may be difficult to sell the benefits of adding FXS to a universal newborn screening panel since it is not a life-threatening condition, as are the metabolic disorders. With all the inherent complications of FXS newborn screening, tremendous educational efforts will be needed to raise public awareness of the disorder and emphasize the benefits of early detection.

As noted by Hill et al., ${ }^{1}$ an alternative to FXS newborn screening is infant screening. Infant screening would be an entirely voluntary process with full informed consent from the family that may alleviate some of the general public's fears about newborn screening because it would allow for more education and give time for informed consent. For example, at the 6- or 9-month well-baby visit, physicians and/or counselors can provide information and educational materials about FXS screening to families. At a subsequent visit, the family can ask questions and choose whether or not to have the screening. Positive results of the screen would be available at the next doctor's visit allowing for early intervention therapies to be initiated. The diagnosis of FXS at around 12 months of age in the infant screening model is a vast improvement over the average current age of diagnosis, which is now $\sim 36$ months. Infant screening would still prevent the "diagnostic odyssey," one of the arguments in favor of newborn screening, because initial parent concerns are usually not raised until around the first year of life. Also, concerns about the negative impact of identifying individuals with FXS and sex chromosome aneuploidies in early infancy on parental bonding would be lessened. Finally, having a voluntary and fully transparent process may make the public more comfortable with such a screening program. One argument against infant screening is that it may not be accessible to all parents, whereas newborn screening would be; however, the risk of missing a few positives may be outweighed by the benefits of allowing families to make the choice of having their child screened for FXS.

In summary, the mutational mechanism and the spectrum of phenotypes associated with FMRI make population screening for FXS more challenging than traditional population screening programs. Given that the vast majority of the general public has never heard of FXS, much less are aware of the complexities of screening and predictive testing of premutation-associated disorders, educational and counseling services need to be developed to inform families of the risks and benefits of the screening. Educational materials need to be delivered at a time and in a manner that allows families to make more informed decisions about screening. The likelihood of success of population screening for FMR1 mutations will greatly improve if the process is voluntary and transparent. Hill et al. ${ }^{1}$ provide an excellent list of suggestions for future research to enable the field to move ahead successfully.

\section{REFERENCE}

1. Hill MK, Archibald AD, Cohen J, et al. A systematic review of population screening for fragile X syndrome. Genet Med 2010;12:xxx-xxx. 